Review Article

\title{
Genomic and Histopathological Tissue Biomarkers That Predict Radiotherapy Response in Localised Prostate Cancer
}

\author{
Anna Wilkins, ${ }^{1,2}$ David Dearnaley, ${ }^{2}$ and Navita Somaiah ${ }^{2,3}$ \\ ${ }^{1}$ Division of Clinical Studies, The Institute of Cancer Research, London SM2 5NG, UK \\ ${ }^{2}$ Division of Radiotherapy and Imaging, The Institute of Cancer Research, London SM2 5NG, UK \\ ${ }^{3}$ Division of Cancer Biology, The Institute of Cancer Research, London SM2 5NG, UK \\ Correspondence should be addressed to Anna Wilkins; anna.wilkins@icr.ac.uk
}

Received 1 April 2015; Accepted 24 May 2015

Academic Editor: Franco M. Buonaguro

Copyright (C) 2015 Anna Wilkins et al. This is an open access article distributed under the Creative Commons Attribution License, which permits unrestricted use, distribution, and reproduction in any medium, provided the original work is properly cited.

\begin{abstract}
Localised prostate cancer, in particular, intermediate risk disease, has varied survival outcomes that cannot be predicted accurately using current clinical risk factors. External beam radiotherapy (EBRT) is one of the standard curative treatment options for localised disease and its efficacy is related to wide ranging aspects of tumour biology. Histopathological techniques including immunohistochemistry and a variety of genomic assays have been used to identify biomarkers of tumour proliferation, cell cycle checkpoints, hypoxia, DNA repair, apoptosis, and androgen synthesis, which predict response to radiotherapy. Global measures of genomic instability also show exciting capacity to predict survival outcomes following EBRT. There is also an urgent clinical need for biomarkers to predict the radiotherapy fraction sensitivity of different prostate tumours and preclinical studies point to possible candidates. Finally, the increased resolution of next generation sequencing (NGS) is likely to enable yet more precise molecular predictions of radiotherapy response and fraction sensitivity.
\end{abstract}

\section{Introduction}

Heterogeneity in tumour biology between prostate tumours results in a varied response to radiotherapy. At present no molecular tissue biomarkers are in routine clinical use to risk-stratify patients with localised prostate cancer. Instead, current management of localised prostate cancer is based upon established clinical risk factors including presenting PSA, clinical or radiological $\mathrm{T}$ (tumour) stage, and the total Gleason score. However, estimates of recurrence and survival vary considerably; for example, in the National Comprehensive Cancer Network (NCCN) intermediate risk group biochemical failure at five years following definitive local therapy varies from $2 \%$ to $70 \%$ [1]. Although new clinical factors have been identified including percentage core positivity and the primary Gleason score [2], there remains an urgent need to incorporate molecular biomarkers predicting radioresistance into treatment decisions. Such biomarkers would enable a personalised prediction of radiotherapy efficacy. If combined with personalised predictors of radiation toxicity including radiogenomic markers [3], both sides of the therapeutic ratio of radiotherapy for localised prostate cancer would be improved.

The lethality of radiotherapy is centred on the creation of chromosomal lesions including DNA double strand breaks (DSB), which are particularly lethal when they cluster in close physical proximity to each other [4]. Cells that are unable to repair this radiation induced DNA damage are likely to undergo programmed cell death via apoptosis or autophagy or alternatively death via mitotic catastrophe $[5$, 6]. Hypoxia has traditionally been viewed as an important contributor to radioresistance as oxygen reacts with damaged DNA bases created by free radicals thus creating a stable adduct and fixing the damage [7]. More recently, the hypoxic state has also been associated with reduced capacity for DNA repair, increasing genomic instability, and creation of a mutator phenotype [8]. Whilst biomarkers of DNA repair and hypoxia have been shown to predict radioresistance, much broader aspects of tumour biology including cell proliferation, apoptosis, and androgen synthesis have been implicated in treatment failure following radiotherapy. All of these offer considerable potential for improving treatment 
precision, for example, with personalised dose escalation or concomitant use of systemic agents such as abiraterone.

Another important radiobiological question at present is the radiotherapy fraction size sensitivity of prostate cancer, as measured by the alpha/beta ratio. An expanding body of evidence points to the alpha/beta ratio of prostate adenocarcinoma being as low as 1.5 [9], suggesting that tumours are more sensitive to fraction size than neighbouring normal tissues. The results of randomised clinical trials testing this hypothesis are currently awaited [10]. However, it is highly likely that the alpha/beta ratio and therefore fraction size sensitivity differ between individual prostate tumours, especially as we know that cellular proliferation, DNA repair, hypoxia, and other relevant biological parameters vary considerably. Although an exciting area of research, once again no molecular biomarkers are in clinical use to assess fraction size sensitivity of tumours prior to radiotherapy treatment.

Recent rapid progress in next generation sequencing techniques offers huge potential for personalisation of radiotherapy treatment, despite some of the required technological expertise being currently beyond the scope of most routine pathology laboratories. Other routinely available histopathological techniques such as immunohistochemistry (IHC) or genomic techniques such as fluorescent in situ hybridisation (FISH), comparative genomic hybridisation $(\mathrm{CGH})$, and polymerase chain reaction (PCR) have identified many candidate biomarkers which with further validation could rapidly enter the clinic.

Molecular biomarker development following prostatectomy has progressed at an accelerated pace compared to following radiotherapy due to limited tissue availability with the latter treatment [11]. Critics suggest that diagnostic biopsies do not represent the true biological heterogeneity within the entire prostate gland. However, as image-guided template biopsies become more commonly used, tumour representation in prostate biopsies continues to increase in accuracy. Furthermore, for the foreseeable future, diagnostic biopsies will continue to be the main tumour tissue available to guide radiotherapy stratification. It is important that the above differences in tissue availability do not hinder the development and validation of predictive biomarkers that distinguish benefit from different local treatments for early prostate cancer as this remains a clinical priority.

This paper aims to review biomarkers predicting radiotherapy response in prostate cancer incorporating genomic signatures and individual candidates as well as biomarkers identified by longer established techniques. It does not address microRNA or biomarkers involved in the diagnosis of prostate cancer or prognostication outside of radiotherapy treatment; these were comprehensively reviewed in a recent paper in this journal [12].

\section{Biomarkers of Radiosensitivity Identified Using Immunohistochemistry (IHC)}

IHC enables direct evaluation of protein expression, which is advantageous as proteins are determinants of cellular function. Recent comprehensive genomic and proteomic work suggests that changes in nucleic acid do not necessarily translate to corresponding changes in protein expression [13]. IHC is a technique that is readily available in routine pathology laboratories; tumour histopathology can be correlated with protein expression; hence, tumour dissection is not required. For bulky prostate tumours, sufficient tissue may be present to construct tissue microarrays which facilitates high throughput analysis [14, 15]; however, in intermediate risk localised prostate tumours this approach has recently been shown to be unfeasible due to inadequate numbers of tumour cells [16].

The Radiation Therapy Oncology Group (RTOG) 8610 and 9202 clinical trials of radiotherapy and varying lengths of androgen deprivation for localised prostate cancer have reported several biomarkers predicting outcome using IHC (Table 1). A consistent prediction of survival outcomes has been shown for some candidates and the second trial has validated earlier findings. For example, Ki-67, a wellestablished marker of cellular proliferation, has consistently predicted biochemical-free survival, local recurrence, and overall survival [17-21]. p53, one of the most commonly mutated tumour suppressor genes with a central role in checkpoint activation, is regulated by the oncogene MDM2. Both genes have shown prediction of prostate cancer outcome in the RTOG studies and elsewhere [17, 22-27]. Low expression of the cyclin dependent kinase inhibitor p16 has also been consistently associated with poor survival outcomes following radiotherapy $[28,29]$. As poor outcomes following surgery are predicted by high expression, p16 is one of very few true predictive biomarkers identified to date [11]. Finally increased expression of COX2, a gene with cell cycle modulatory effects as well as antiapoptotic, proangiogenic, and proliferative effects via prostaglandin E2 [30, 31], has also been repeatedly associated with poor survival outcomes [32].

Pollack et al. recently modelled the risk of distant metastases using expression of the above 5 candidates plus the apoptotic proteins $\mathrm{Bcl}-2$ and $\mathrm{Bax}$ with competing risks hazard regression, adjusting for age, PSA, the Gleason score, T-stage, and treatment [33]. The resulting model included 4 tissue biomarkers (Ki-67, MDM2, p16, and COX2) and showed a concordance index of 0.77 versus 0.70 without molecular biomarkers, meaning a relative improvement in prediction of distant metastases of $10 \%$. This "immunopanel" is the first known multiplex panel of biomarkers developed using IHC to date in prostate cancer.

The role of hypoxia markers in prognostication following radiotherapy is more controversial. VEGF and HIF1-alpha were not included in the RTOG modelling of risk of distant metastases because an earlier RTOG study failed to demonstrate a significant association of VEGF with any survival outcomes following radiotherapy [34]. However, in two British studies of VEGF and HIF1-alpha, increased expression independently predicted biochemical recurrence $[35,36]$. Furthermore, lactate dehydrogenase (LDH), a marker of anaerobic metabolism and an indirect marker of hypoxia, has also been associated with inferior radiotherapy response [37]. Osteopontin (OPN) is a small integrin-binding ligand $\mathrm{N}$ linked glycoprotein (SIBLING) that is thought to be induced by hypoxia [38]. OPN has been associated with reduced 
TABLE 1: Predictive tissue biomarkers for radiotherapy response identified using immunohistochemistry (IHC).

\begin{tabular}{|c|c|c|c|}
\hline \multicolumn{4}{|c|}{ Tissue biomarkers for radiotherapy response identified using IHC } \\
\hline Marker & Function & Technique & IHC cut point used \\
\hline p53 $[22,23,25,27]$ & Cell cycle checkpoints & IHC & $\begin{array}{l}0 \text { versus } 1-4^{*}[22], \leq 30 \% \text { nuclei versus }>30 \% \text { nuclei }[23],<20 \% \\
\text { nuclei versus } \geq 20 \% \text { nuclei }[25] \text {, and } 0 \text { versus } 1 \text { versus } 2-4[27]\end{array}$ \\
\hline p16 $[28,29]$ & Cell cycle checkpoints & IHC & $\leq 25 \%$ versus $>25 \%[28], \leq 81.3 \%$ versus $>81.3 \%[29]$ \\
\hline Rb1 [28] & Cell cycle checkpoints & IHC & $\leq 20 \%$ versus $>20 \%$ \\
\hline MDM2 $[17,24]$ & Cell cycle checkpoints & $\mathrm{IHC}$ & $\leq 184$ versus $>184$ AU (IA) $[17], \leq 3 \%$ versus $>3 \%$ ACIS $[24]$ \\
\hline Ki67 [17-21] & Cell proliferation & IHC & $\begin{array}{l}\leq 11.3 \% \text { nuclei versus }>11.3 \% \text { nuclei }[17], \text { SI } \leq 3.5 \% \text { versus }>3.5 \% \\
{[18,19], \text { continuous and } \text { SI } \leq 7.1 \% \text { versus }>7.1 \%[20] \text {, and SI }} \\
<6.2 \% \text { versus } \geq 6.2 \%[21]\end{array}$ \\
\hline PKA [39] & Cell proliferation & IHC & $\begin{array}{l}\text { Manual: } 0,1 \text {, and } 2 \text { versus } 3 \text { and } 0,1 \text { versus } 2,3^{*} \text { IA: median of } \\
111.8 \text {, Q3 of } 128.0 \text { and } 135.5\end{array}$ \\
\hline $\begin{array}{l}\text { STAT3 (activated) } \\
{[40]}\end{array}$ & $\begin{array}{l}\text { Cell } \\
\text { proliferation/apoptosis }\end{array}$ & IHC & Continuous and ACIS $\leq 29 \%$ versus $>29 \%$ \\
\hline Her2/neu [41] & Growth receptor & IHC & Membrane positivity $\leq 10 \%$ versus $>10 \%$ \\
\hline EGFR [42] & Growth receptor & IHC & Negative versus weak or strong membranous staining \\
\hline Bcl2 $[22,23,43]$ & Apoptosis & IHC & $\begin{array}{l}\text { Nil versus any cytoplasmic staining }[22,23], \leq 20 \% \text { versus }>20 \% \\
\text { cytoplasmic staining }[43]\end{array}$ \\
\hline $\operatorname{Bax}[44]$ & Apoptosis & IHC & $\begin{array}{l}\text { Greater or lesser cytoplasm staining intensity relative to normal } \\
\text { prostate }\end{array}$ \\
\hline E-cadherin $[15]$ & Cell adhesion & PCR array, IHC validation & Absent or weak $(0 / 1+)$ versus moderate or strong $(2+/ 3+)$ \\
\hline COX2 [32] & Prostaglandin synthesis & IHC & $134 \mathrm{AU}$ (median) and continuous variable [32] \\
\hline LDH5 [37] & $\begin{array}{l}\text { Anaerobic metabolism } \\
\text { and hypoxia }\end{array}$ & IHC & $\begin{array}{l}<50 \% \text { cytoplasmic expression and/or }<10 \% \text { nuclear expression } \\
\text { versus }>50 \% \text { and }>10 \%\end{array}$ \\
\hline HIFla $[35,42]$ & Hypoxia & IHC & $\begin{array}{l}0 \% \text { versus }<1 \% \text { versus } 1-10 \% \text { versus } 10-33 \% \text { versus } 34-67 \% \\
\text { versus }>67 \% \text { cytoplasmic staining }[35], \leq 50 \% \text { versus }>50 \% \\
\text { nuclear and cytoplasm staining }[42]\end{array}$ \\
\hline VEGF $[35,36]$ & Hypoxia & IHC & $\begin{array}{l}0 \% \text { versus }<1 \% \text { versus } 1-10 \% \text { versus } 10-50 \% \text { versus }>50 \% \\
\text { cytoplasmic staining [35], IRS score }{ }^{*} 0-4 \text { versus } 5-8[36]\end{array}$ \\
\hline DNA PKcs [45] & NHEJ & IHC & Nil versus any nuclear staining \\
\hline
\end{tabular}

NHEJ: nonhomologous end joining; PCR: polymerase chain reaction; IA: image analysis; IRS: immunoreactive score; AU: arbitrary units; ACIS: automated cellular imaging system; ${ }^{*}$ cut point refers to semiquantitative scoring system incorporating staining intensity and percentage of tumour cells positive; SI: staining index.

survival times in prostate cancer [38], however, was only significant in predicting radiotherapy response on univariate analysis, not when modelling adjusted for other clinical factors [35]. A study of plasma OPN levels in localised prostate cancer indicated that OPN levels did not change in response to radiotherapy [46].

There are several possible reasons for conflicting data regarding hypoxic biomarkers and prediction of radiotherapy response. These include differences in the size of patient cohorts, NCCN risk group, and IHC cut points used to determine high expression of VEGF and HIF1-alpha. Furthermore, our understanding of how hypoxia impacts DNA repair is evolving rapidly. Recent studies suggest that hypoxia induces downregulation of proteins within the DNA double strand break repair pathways of homologous recombination (HR) and nonhomologous end joining (NHEJ) [47-49]. This has implications for radiosensitivity and also provides a mechanism for hypoxia inducing a mutator phenotype as DNA repair downregulation could permit survival and subsequent clonal selection of unrepaired unstable mutant tumour cells [8]. Further work to clarify the role of hypoxic markers in treatment stratification would be of considerable value.
With regard to DNA repair, error prone NHEJ operates in all phases of the cell cycle to repair DNA DSB; DNA PKcs has a key role in NHEJ by forming a synaptic complex bringing the free broken ends of DNA together with other ligating enzymes. Nuclear expression of DNA PKcs using IHC showed an independent association with biochemical recurrence after radiotherapy. However, other NHEJ proteins, also evaluated with IHC, such as Ku70, Ku80, and XRCC4 were not predictive of relapse [45].

The TMPRSS2/ERG fusion is an important cellular rearrangement occurring in $50 \%$ of localised prostate tumours and ERG protein expression using IHC has been shown to be a robust surrogate for detecting the gene fusion [50]. Preclinical studies have suggested that the gene fusion may be a biomarker of inferior double stranded DNA break repair capacity with important clinical implications [51]. However, the gene fusion was not prognostic for recurrence after radiotherapy when assessed with either IHC or CGH suggesting that it does not affect prostate tumour radiosensitivity [16] (Table 3).

There is thought to be direct cross talk between the EGFR cellular proliferation pathway and DNA repair. This provides 
TABLE 2: Predictive tissue biomarkers for radiotherapy response identified using genomic techniques.

\begin{tabular}{lcc}
\hline Marker/signature & Function & Technique \\
\hline DNA ploidy [52] & Genomic instability & $\begin{array}{c}\text { Image analysis (Feulgen) and } \\
\text { DNA/protein flow cytometry } \\
\text { Static DNA cytometry }\end{array}$ \\
Nuclear DNA content [53] & Genomic instability & Array CGH validated by FISH \\
\hline c-myc [54] & Cell proliferation & Array CGH validated by FISH \\
\hline PTEN [54] & Cell survival & PCR array, IHC validation \\
\hline E-cadherin [15] & Cell adhesion & Array CGH \\
NKX3.1 [55] & Androgen related homeobox gene, DNA & Array CGH \\
NBN [56] & repair & Array CGH \\
\hline StAR [57] & DNA damage response & Array CGH \\
\hline HSD17B2 [57] & Androgen synthesis & Androgen synthesis \\
\hline Cell cycle progression score [58] & Cell cycle progression & RT-PCR (RNA expression) \\
\hline CAN_RF [59] & Genomic and microenvironment & Array CGH, intraglandular hypoxia with \\
\end{tabular}

FISH: fluorescent in situ hybridisation; CGH: comparative genomic hybridisation; RT-PCR: reverse transcription polymerase chain reaction.

a possible biological rationale for the observed correlation between high EGFR expression and poor prognosis following prostate radiotherapy [42]. However, the aetiology of poor survival outcomes with increased EGFR expression is likely to be multifactorial and includes increased cellular proliferation. Protein kinase A type 1 and STAT3 also function in cell proliferation and malignant transformation and have been studied in the RTOG trials where overexpression has been associated with poorer outcomes [39, 40]. However, STAT3 expression only correlated with distant metastases and not with other survival outcomes such as local failure. For protein kinase A, overexpression was associated with a diminished response to long term androgen deprivation therapy (LTAD) and radiation, relative to short term androgen deprivation and radiotherapy suggesting that these patients require alternative treatment escalation to LTAD.

The antiapoptotic protein $\mathrm{Bcl}-2$ and proapoptotic Bax both have key roles in determining cell fate following radiotherapy. Independent prediction of survival outcomes has been demonstrated in some $[22,23,43]$, but not all $[44,60]$, studies to date and further work to clarify their prognostic role is needed. Androgen deprivation is known to cause apoptotic cell death so different use of androgen deprivation within treatment arms of RTOG 8610 and 9202 is likely to have impacted predictive outcomes [44]. Some of the observed discrepancies may have arisen due to high numbers of patients with locally advanced versus early prostate cancer, a lack of standardised IHC protocol and antibody, as well as use of different cut points defining high or low expression between studies.

Identifying biomarkers of radiotherapy fraction size sensitivity is another area of unmet need. There is a tight association between proliferative indices and fraction size sensitivity of normal tissues [61]. Normal tissues that respond early (within days of radiotherapy) have high proliferative indices and low sensitivity to fraction size and vice versa for those that respond late (years later). The association is so tight as to offer clues to mechanisms [62-64]. Published literature also suggests that the choice of DNA DSB repair pathway (homologous recombination (HR) versus NHEJ) between rapidly proliferating and slowly proliferating cells may influence fraction size sensitivity. Using in vitro clonogenic assays rodent cell lines with defects in NHEJ showed loss of fraction size sensitivity $[65,66]$. In addition, IHC on in vivo irradiated human skin showed that a 10-fold increase in the use of HR to repair radiation-induced DNA DSB towards the end of radiotherapy correlated with loss of fractionation sensitivity seen clinically [67]. On average, prostate tumours are thought to have a low alpha/beta ratio and hence are sensitive to both fraction size and total dose of radiation [9]. However, biological heterogeneity means that fraction size sensitivity is likely to vary between prostate tumours and therefore there is a need to identify tissue biomarkers to guide individualised fractionation.

\section{Biomarkers of Radiosensitivity Identified Using Genomic Techniques}

3.1. Fluorescent In Situ Hybridisation (FISH). Use of a fluorogen with FISH, rather than a chromogen as in IHC, means that interpreting expression can be more straightforward than with IHC [68]. In addition, multiple fluorophores can be combined on a single slide which is particularly advantageous with the limited tissue available from pre-radiotherapy biopsies [69]. FISH is routinely available in histopathology laboratories where it is particularly useful for confirming HER2 status in breast tumours and therefore the technique offers considerable potential for development of predictive biomarkers.

FISH has been used to demonstrate a role for biomarkers of cell proliferation such as PTEN and c-myc in predicting radiotherapy response. Loss of the tumour suppressor gene PTEN and amplification of the oncogene c-myc have both been associated with inferior outcomes following radiotherapy [54] (Table 2). In combination, these biomarkers were more strongly associated with increased biochemical recurrence than either in isolation. 
TABLE 3: Negative predictive studies in EBRT.

\begin{tabular}{|c|c|c|c|}
\hline Marker & Function & Technique & IHC cut point used \\
\hline VEGF [34] & Hypoxia & IHC & $0-1$ versus $2-3$ cytoplasmic staining intensity \\
\hline Bcl-2 $[44,60,70]$ & Apoptosis & IHC & $\begin{array}{l}\text { Nil versus any cytoplasmic staining }[44,60] \text {, } \\
<10 \% \text { versus }>10 \% \text { cell staining }[70]\end{array}$ \\
\hline $\operatorname{Bax}[60]$ & Apoptosis & IHC & $\begin{array}{l}\text { Greater or lesser cytoplasm staining intensity } \\
\text { relative to normal prostate }\end{array}$ \\
\hline AR [15] & Androgen receptor & PCR array & \\
\hline PCA3 [15] & Prostate marker & PCR array & \\
\hline PTEN [15] & Cell survival & PCR array & \\
\hline EZH2 [15] & Transcriptional control & PCR array & \\
\hline EGFR [15] & Growth receptor & PCR array & \\
\hline PSMA [15] & Prostate marker & PCR array & \\
\hline MSMB [15] & Tumour suppression & PCR array & \\
\hline CAG repeats $[71]$ & & $\begin{array}{l}\text { Genotyping } \\
\text { (PCR) }\end{array}$ & \\
\hline $\begin{array}{l}\text { CYP3A4 polymorphisms } \\
{[72]}\end{array}$ & & Genotyping & \\
\hline $\begin{array}{l}\text { TMPRSS2/ERG (or ETV1) } \\
\text { [16] }\end{array}$ & $\begin{array}{l}\text { Androgen regulated/cell } \\
\text { proliferation gene fusion }\end{array}$ & $\begin{array}{l}\text { Array CGH, } \\
\text { IHC }\end{array}$ & Any positive staining versus negative \\
\hline Osteopontin [46] & $\begin{array}{l}\text { SIBLING, tumour } \\
\text { associated protein }\end{array}$ & ELISA & \\
\hline $\mathrm{Ku} 70$ and $\mathrm{Ku} 80$ [45] & NHEJ & IHC & $\begin{array}{l}\mathrm{Ku} 70 \leq 50 \% \text { versus }>50 \% \text { nuclear staining, } \\
\mathrm{Ku} 80 \leq 60 \% \text { versus }>60 \% \text { nuclear staining }\end{array}$ \\
\hline MRE11 [56] & DNA damage response & Array CGH & \\
\hline RAD50 [56] & DNA damage response & Array CGH & \\
\hline ATM $[56]$ & DNA damage response & Array CGH & \\
\hline ATR $[56]$ & DNA damage response & Array CGH & \\
\hline PRKDC (DNA PKcs) [56] & NHEJ & Array CGH & \\
\hline
\end{tabular}

SIBLING: small integrin-binding ligand: N-linked glycoprotein.

3.2. Polymerase Chain Reaction (PCR) Array. PCR array involves synthesis and amplification of complimentary DNA (cDNA) prior to expression profiling. Although amplification can introduce bias, this multiplex technique is particularly useful when tissue and hence nucleic acid quantity is limited. A reduction in mRNA (messenger RNA) of the cell-cell adhesion molecule E-cadherin has recently been associated with poor outcome after radiotherapy, but not after primary androgen deprivation therapy alone using PCR array [15]. The authors validated the predictive ability of E-cadherin in an independent dataset by demonstrating that reduced protein expression using IHC was significantly and independently associated with early biochemical recurrence. A number of other candidates were simultaneously assessed including previously discussed EGFR and PTEN, as well as EZH2, PSMA, and MSMB, all of which have shown prognostic ability following surgery; however, they were non-prognostic after radiotherapy in this study. However, the study cohort size was modest at 60 patients which may have contributed to negative findings [15]. The fact that AR also showed no prognostic ability after EBRT illustrates the differences in tumour biology between castration resistant and castration sensitive tumours.
3.3. Comparative Genomic Hybridisation (CGH). CGH differs from Reverse Transcription Polymerase Chain Reaction (RT-PCR) in that it measures DNA copy number variations rather than messenger RNA expression. It thus enables a measure of genomic instability and can calculate the percentage of genomic alteration (PGA) per tumour sample. Today CGH arrays are able to evaluate global copy number variations with as little as $100 \mathrm{ng}$ of DNA [73]. This is highly relevant to localised prostate tumours treated with radiotherapy, not only because there is limited tissue available but also because progression of prostate cancer is known to be characterised by increased chromosomal and subchromosomal alterations characteristic of genomic instability [73]. Some of the earliest prognostic molecular biomarkers identified over two decades ago were based on detection of genomic instability in the form of polyploidy or nondiploidy assessed using flow cytometry and nuclear DNA content measured by static DNA cytometry $[52,53]$.

Using CGH, copy number loss of several novel biomarkers with diverse functions has been proposed, as well as further validation of previously identified candidates including PTEN [54] (Table 3). These include two genes involved in androgen synthesis, namely, androgen synthesis genes 
steroidogenic acute regulatory protein (StAR) and hydroxysteroid (17-beta) dehydrogenase 2 HSD17B2 [57]. NKX3.1 is a tumour suppressor gene with a role in prostate stem cell maintenance. It interacts with topoisomerase I and is thought to facilitate recruitment of phosphorylated ATM and gamma H2AX to sites of DNA double strand breaks, both highly relevant to the DNA damage response [55]. NKX3.1 allelic loss alone independently predicted failure from imageguided radiotherapy (IGRT) in a model adjusting for relevant clinical parameters, androgen treatment, radiotherapy dose, and PGA [55]. When allelic gain in c-myc was combined with NKX3.1 loss, the combination showed further predictive capacity [55]. Nibrin (NBN), also known as Nijmegen Breakage Syndrome- (NBS-) 1, forms part of the MRN complex which is central to initiation of the DNA damage response (DDR). In a study of 6 important genes in the DDR (also including MRE11A, RAD50, ATM, ATR, and PRKDC) NBN gain was the only copy number variation significantly predicting biochemical recurrence after IGRT [56]. As it did not predict outcome after radical prostatectomy, NBN may have a role as a predictive biomarker guiding local treatment decisions.

3.4. RNA Expression Profiles. A number of RNA expression signatures have been proposed to risk-stratify in localised prostate cancer $[74,75]$. The majority have not been evaluated in a radiotherapy cohort due to inadequate tissue quantity, although the oncotype DX for prostate has been tested on needle biopsy specimens [76]. The cell cycle progression score is a 31-gene signature based on RNA expression which was developed using quantitative RT-PCR [77]. The 31 genes were selected from a larger panel of 126 candidate genes known to be involved in cell cycle progression within the Gene Expression Omnibus database. The score includes genes with central roles in DNA repair such as RAD51. Initially developed using radical prostatectomy and TURP specimens [77], the signature has subsequently shown significant prediction of biochemical recurrence following image-guided radiotherapy on multivariate analysis that adjusted for known clinical predictive factors [58].

3.5. DNA Signatures. The first known DNA based signature to predict recurrence after EBRT has recently been reported [59] and was developed in a radiotherapy cohort by the use of a customised array to detect copy number alterations together with measurement of partial oxygen pressure using an intraglandular piezoelectrode. Four unique genomic subtypes were identified using unsupervised hierarchical clustering. Information on PGA and hypoxia was then integrated into the genomic subtypes. Finally, supervised machine learning was used to develop a 100-loci 276-gene DNA signature which was validated in a surgically treated cohort. The new signature outperformed a clinical model and 23 previously published RNA signatures in predicting biochemical relapse-free survival. Intriguingly several genes involved in lipid biology were included in the signature; the association of the local cholesterol metabolism of prostate tumours with disease progression has been demonstrated previously [78].
3.6. Next Generation Sequencing. Next generation sequencing (NGS) offers enormous potential for personalisation of treatment. It enables assessment of genomic events usually affecting more than $1 \mathrm{kbp}$ such as structural copy number alterations and chromosomal rearrangements including translocations, inversions, and recombination events. In addition, the powerful resolution of NGS also enables detection of events affecting less than $1 \mathrm{kbp}$ such as substitutions, insertions, and deletions [79]. To our knowledge, biomarker signatures using NGS predicting response to radiotherapy are not yet available, and limited tissue availability may be an explanation for this. However, this is likely to change soon with studies involving combined DNA, RNA, and epigenetic analyses ongoing as part of the International Cancer Genome Consortium and The Cancer Genome Atlas [59]. NGS technology continues to evolve rapidly and recently targeted DNA sequencing of prostate tumours using the Illumina platform has been possible with as little as $30 \mathrm{ng}$ of DNA [80] and using the PGM Ion Torrent platform with as little as $6 \mathrm{ng}$ [81].

\section{Conclusion}

Fundamental aspects of cancer biology including DNA repair and hypoxia are intimately related to the efficacy of radiotherapy. It is therefore not surprising that over recent decades a number of promising tissue biomarkers have been developed using a range of molecular techniques. Whilst the majority of biomarker candidates are protein markers developed using IHC, markers of genomic instability using more quantitative techniques have shown excellent prognostic capability. Validation of these biomarkers is a priority so that the added benefit to standard clinical parameters can be clearly quantified and existing inconsistencies resolved. Development of predictive biomarkers that differentiate benefit from different local treatments and active surveillance would further enhance personalised management of early prostate cancer. Challenges include the need for standardised reproducible protocols and antibodies for IHC, together with the technical limitations of using very small biopsies for genomic techniques. However, technology continues to advance rapidly and the potential for molecular biomarkers to improve prediction of both sides of the therapeutic ratio of radiotherapy for localised prostate cancer is hugely promising.

\section{Conflict of Interests}

The authors declare that there is no conflict of interests regarding the publication of this paper.

\section{Acknowledgments}

The authors acknowledge NHS funding to the NIHR Biomedical Research Centre at The Royal Marsden and The Institute of Cancer Research (ICR), London. Anna Wilkins is supported by a Cancer Research UK Ph.D. Training Fellowship at the CRUK Centre at The Institute of Cancer Research and The Royal Marsden; David Dearnaley is Joint Lead Applicant of 
Cancer Research UK Programme Grant A19727 and Principal Investigator of CRUK (BIDD Project Award) A12518. Navita Somaiah has a Clinician Scientist Fellowship supported by ICR/CRUK funding.

\section{References}

[1] G. D. Grossfeld, D. M. Latini, D. P. Lubeck et al., "Predicting disease recurrence in intermediate and high-risk patients undergoing radical prostatectomy using percent positive biopsies: results from CaPSURE," Urology, vol. 59, no. 4, pp. 560-565, 2002.

[2] Z. S. Zumsteg, D. E. Spratt, I. Pei et al., "A new risk classification system for therapeutic decision making with intermediate-risk prostate cancer patients undergoing dose-escalated externalbeam radiation therapy," European Urology, vol. 64, no. 6, pp. 895-902, 2013.

[3] C. West, B. S. Rosenstein, J. Alsner et al., "Establishment of a radiogenomics consortium," International Journal of Radiation Oncology Biology Physics, vol. 76, no. 5, pp. 1295-1296, 2010.

[4] D. T. Goodhead, "Energy deposition stochastics and track structure: what about the target?" Radiation Protection Dosimetry, vol. 122, no. 1-4, pp. 3-15, 2006.

[5] R. C. Taylor, S. P. Cullen, and S. J. Martin, "Apoptosis: controlled demolition at the cellular level," Nature Reviews Molecular Cell Biology, vol. 9, no. 3, pp. 231-241, 2008.

[6] K. Chu, N. Teele, M. W. Dewey, N. Albright, and W. C. Dewey, "Computerized video time lapse study of cell cycle delay and arrest, mitotic catastrophe, apoptosis and clonogenic survival in irradiated 14-3-3sigma and CDKN1A (p21) knockout cell lines," Radiation Research, vol. 162, no. 3, pp. 270-286, 2004.

[7] K. R. Luoto, R. Kumareswaran, and R. G. Bristow, "Tumor hypoxia as a driving force in genetic instability," Genome Integrity, vol. 4, no. 1, article 5, 2013.

[8] R. Bristow, A. Berlin, and A. Dal Pra, "An arranged marriage for precision medicine: hypoxia and genomic assays in localized prostate cancer radiotherapy," The British Journal of Radiology, vol. 87, no. 1035, 2014.

[9] D. J. Brenner and E. J. Hall, "Fractionation and protraction for radiotherapy of prostate carcinoma," International Journal of Radiation Oncology, Biology, Physics, vol. 43, no. 5, pp. 1095-1101, 1999.

[10] D. Dearnaley, I. Syndikus, G. Sumo et al., "Conventional versus hypofractionated high-dose intensity-modulated radiotherapy for prostate cancer: preliminary safety results from the $\mathrm{CHHiP}$ randomised controlled trial," The Lancet Oncology, vol. 13, no. 1, pp. 43-54, 2012.

[11] V. J. Gnanapragasam, "Molecular markers to guide primary radical treatment selection in localized prostate cancer," Expert Review of Molecular Diagnostics, vol. 14, no. 7, pp. 871-881, 2014.

[12] T. Sequeiros, M. García, M. Montes et al., "Molecular markers for prostate cancer in formalin-fixed paraffin-embedded tissues," BioMed Research International, vol. 2013, Article ID 283635, 15 pages, 2013.

[13] B. Zhang, J. Wang, X. Wang et al., "Proteogenomic characterization of human colon and rectal cancer," Nature, vol. 513, no. 7518, pp. 382-387, 2014.

[14] F. McCarthy, N. Dennis, P. Flohr, S. Jhavar, C. Parker, and C. S. Cooper, "High-density tissue microarrays from prostate needle biopsies," Journal of Clinical Pathology, vol. 64, no. 1, pp. 88-90, 2011.
[15] N. Kachroo, A. Y. Warren, and V. J. Gnanapragasam, "Multitranscript profiling in archival diagnostic prostate cancer needle biopsies to evaluate biomarkers in non-surgically treated men," BMC Cancer, vol. 14, no. 1, article 673, 2014.

[16] A. D. Pra, E. Lalonde, J. Sykes et al., "TMPRSS2-ERG status is not prognostic following prostate cancer radiotherapy: implications for fusion status and DSB repair," Clinical Cancer Research, vol. 19, no. 18, pp. 5202-5209, 2013.

[17] L.-Y. Khor, K. Bae, R. Paulus et al., "MDM2 and Ki-67 predict for distant metastasis and mortality in men treated with radiotherapy and androgen deprivation for prostate cancer: RTOG 92-02," Journal of Clinical Oncology, vol. 27, no. 19, pp. 3177-3184, 2009.

[18] D. Cowen, P. Troncoso, V. S. Khoo et al., "Ki-67 staining is an independent correlate of biochemical failure in prostate cancer treated with radiotherapy," Clinical Cancer Research, vol. 8, no. 5, pp. 1148-1154, 2002.

[19] R. Li, K. Heydon, M. E. Hammond et al., "Ki-67 staining index predicts distant metastasis and survival in locally advanced prostate cancer treated with radiotherapy: an analysis of patients in Radiation Therapy Oncology Group Protocol 8610," Clinical Cancer Research, vol. 10, no. 12, part 1, pp. 4118-4124, 2004.

[20] A. Pollack, M. Desilvio, L.-Y. Khor et al., "Ki-67 staining is a strong predictor of distant metastasis and mortality for men with prostate cancer treated with radiotherapy plus androgen deprivation: Radiation Therapy Oncology Group trial 92-02," Journal of Clinical Oncology, vol. 22, no. 11, pp. 2133-2140, 2004.

[21] B. Verhoven, Y. Yan, M. Ritter et al., "Ki-67 is an independent predictor of metastasis and cause-specific mortality for prostate cancer patients treated on Radiation Therapy Oncology Group (RTOG) 94-08," International Journal of Radiation Oncology Biology Physics, vol. 86, no. 2, pp. 317-323, 2013.

[22] R. Vergis, C. M. Corbishley, K. Thomas et al., "Expression of Bcl-2, p53, and MDM2 in localized prostate cancer with respect to the outcome of radical radiotherapy dose escalation," International Journal of Radiation Oncology, Biology, Physics, vol. 78, no. 1, pp. 35-41, 2010.

[23] D. S. Scherr, E. D. Vaughan Jr., J. Wei et al., "bcl-2 and p53 expression in clinically localized prostate cancer predicts response to external beam radiotherapy," Journal of Urology, vol. 162, no. 1, pp. 12-17, 1999.

[24] L.-Y. Khor, M. DeSilvio, T. Al-Saleem et al., "MDM2 as a predictor of prostate carcinoma outcome: an analysis of Radiation Therapy Oncology Group protocol 8610," Cancer, vol. 104, no. 5, pp. 962-967, 2005.

[25] D. J. Grignon, R. Caplan, F. H. Sarkar et al., "p53 status and prognosis of locally advanced prostatic adenocarcinoma: a study based on RTOG 8610," Journal of the National Cancer Institute, vol. 89, no. 2, pp. 158-165, 1997.

[26] M. Che, M. DeSilvio, A. Pollack et al., "Prognostic value of abnormal p53 expression in locally advanced prostate cancer treated with androgen deprivation and radiotherapy: a study based on RTOG 9202," International Journal of Radiation Oncology Biology Physics, vol. 69, no. 4, pp. 1117-1123, 2007.

[27] M. A. Ritter, K. W. Gilchrist, M. Voytovich, R. J. Chappell, and B. M. Verhoven, "The role of p53 in radiation therapy outcomes for favorable-to-intermediate-risk prostate cancer," International Journal of Radiation Oncology, Biology, Physics, vol. 53, no. 3, pp. 574-580, 2002.

[28] A. Chakravarti, K. Heydon, C. L. Wu et al., "Loss of p16 expression is of prognostic significance in locally advanced 
prostate cancer: an analysis from the Radiation Therapy Oncology Group protocol 86-10," Journal of Clinical Oncology, vol. 21, no. 17, pp. 3328-3334, 2003.

[29] A. Chakravarti, M. DeSilvio, M. Zhang et al., "Prognostic value of p16 in locally advanced prostate cancer: a study based on Radiation Therapy Oncology Group protocol 9202," Journal of Clinical Oncology, vol. 25, no. 21, pp. 3082-3089, 2007.

[30] A. Kirschenbaum, X.-H. Liu, S. Yao, and A. C. Levine, “The role of cyclooxygenase-2 in prostate cancer," Urology, vol. 58, no. 2, supplement 1, pp. 127-131, 2001.

[31] Y.-M. Kim, Y. K. Shin, H. J. Jun, S. Y. Rha, and H. Pyo, "Systematic analyses of genes associated with radio sensitizing effect by celecoxib, a specific cyclooxygenase-2 inhibitor," Journal of Radiation Research, vol. 52, no. 6, pp. 752-765, 2011.

[32] L.-Y. Khor, K. Bae, A. Pollack et al., "COX-2 expression predicts prostate-cancer outcome: analysis of data from the RTOG 92-02 trial," The Lancet Oncology, vol. 8, no. 10, pp. 912-920, 2007.

[33] A. Pollack, J. J. Dignam, D. A. Diaz et al., "A tissue biomarkerbased model that identifies patients with a high risk of distant metastasis and differential survival by length of androgen deprivation therapy in RTOG protocol 92-02," Clinical Cancer Research, vol. 20, no. 24, pp. 6379-6388, 2014.

[34] L. Pan, S. Baek, P. R. Edmonds et al., "Vascular endothelial growth factor (VEGF) expression in locally advanced prostate cancer: secondary analysis of Radiation Therapy Oncology Group (RTOG) 8610," Radiation Oncology, vol. 8, no. 1, article 100, 2013.

[35] R. Vergis, C. M. Corbishley, A. R. Norman et al., "Intrinsic markers of tumour hypoxia and angiogenesis in localised prostate cancer and outcome of radical treatment: a retrospective analysis of two randomised radiotherapy trials and one surgical cohort study," The Lancet Oncology, vol. 9, no. 4, pp. 342-351, 2008.

[36] M. M. L. Green, C. T. Hiley, J. H. Shanks et al., "Expression of vascular endothelial growth factor (VEGF) in locally invasive prostate cancer is prognostic for radiotherapy outcome," International Journal of Radiation Oncology Biology Physics, vol. 67, no. 1, pp. 84-90, 2007.

[37] M. I. Koukourakis, A. Giatromanolaki, M. Panteliadou et al., "Lactate dehydrogenase 5 isoenzyme overexpression defines resistance of prostate cancer to radiotherapy," British Journal of Cancer, vol. 110, no. 9, pp. 2217-2223, 2014.

[38] D. T. Denhardt and X. Guo, "Osteopontin: a protein with diverse functions," The FASEB Journal, vol. 7, no. 15, pp. 1475-1482, 1993.

[39] L.-Y. Khor, K. Bae, T. Al-Saleem et al., "Protein kinase A RI$\alpha$ predicts for prostate cancer outcome: analysis of Radiation Therapy Oncology Group trial 86-10," International Journal of Radiation Oncology, Biology, Physics, vol. 71, no. 5, pp. 1309-1315, 2008.

[40] J. F. Torres-Roca, M. DeSilvio, L. B. Mora et al., "Activated STAT3 as a correlate of distant metastasis in prostate cancer: a secondary analysis of Radiation Therapy Oncology Group 8610," Urology, vol. 69, no. 3, pp. 505-509, 2007.

[41] A. Fosså, W. Lilleby, S. D. Fosså, G. Gaudernack, G. Torlakovic, and A. Berner, "Independent prognostic significance of Her2 oncoprotein expression in pN0 prostate cancer undergoing curative radiotherapy," International Journal of Cancer, vol. 99, no. 1, pp. 100-105, 2002.

[42] D. C. Weber, J.-C. Tille, C. Combescure et al., "The prognostic value of expression of HIF1 $\alpha$, EGFR and VEGF-A, in localized prostate cancer for intermediate- and high-risk patients treated with radiation therapy with or without androgen deprivation therapy," Radiation Oncology, vol. 7, no. 1, article 66, 2012.

[43] A. Pollack, D. Cowen, P. Troncoso et al., "Molecular markers of outcome after radiotherapy in patients with prostate carcinoma: Ki-67, bcl-2, bax, and bcl-x," Cancer, vol. 97, no. 7, pp. 1630-1638, 2003.

[44] L. Y. Khor, J. Moughan, T. Al-Saleem et al., "Bcl-2 and bax expression predict prostate cancer outcome in men treated with androgen deprivation and radiotherapy on Radiation Therapy Oncology Group protocol 92-02," Clinical Cancer Research, vol. 13, no. 12, pp. 3585-3590, 2007.

[45] P. Bouchaert, S. Guerif, C. Debiais, J. Irani, and G. Fromont, "DNA-PKcs expression predicts response to radiotherapy in prostate cancer," International Journal of Radiation Oncology Biology Physics, vol. 84, no. 5, pp. 1179-1185, 2012.

[46] J. W. Thoms, A. dal Pra, P. H. Anborgh et al., "Plasma osteopontin as a biomarker of prostate cancer aggression: relationship to risk category and treatment response," British Journal of Cancer, vol. 107, no. 5, pp. 840-846, 2012.

[47] A. X. Meng, F. Jalali, A. Cuddihy et al., "Hypoxia down-regulates DNA double strand break repair gene expression in prostate cancer cells," Radiotherapy and Oncology, vol. 76, no. 2, pp. 168176, 2005.

[48] R. Kumareswaran, O. Ludkovski, A. Meng, J. Sykes, M. Pintilie, and R. G. Bristow, "Chronic hypoxia compromises repair of DNA double-strand breaks to drive genetic instability," Journal of Cell Science, vol. 125, no. 1, pp. 189-199, 2012.

[49] N. Chan, M. Koritzinsky, H. Zhao et al., "Chronic hypoxia decreases synthesis of homologous recombination proteins to offset chemoresistance and radioresistance," Cancer Research, vol. 68, no. 2, pp. 605-614, 2008.

[50] S. M. Falzarano, M. Zhou, P. Carver et al., "ERG gene rearrangement status in prostate cancer detected by immunohistochemistry," Virchows Archiv, vol. 459, no. 4, pp. 441-447, 2011.

[51] T. A. Swanson, S. A. Krueger, S. Galoforo et al., “TMPRSS2/ERG fusion gene expression alters chemo- and radio-responsiveness in cell culture models of androgen independent prostate cancer," The Prostate, vol. 71, no. 14, pp. 1548-1558, 2011.

[52] A. Pollack, G. K. Zagars, A. K. El-Naggar, M. D. Gauwitz, and N. H. A. Terry, "Near-diploidy: a new prognostic factor for clinically localized prostate cancer treated with external beam radiation therapy," Cancer, vol. 73, no. 7, pp. 1895-1903, 1994.

[53] J. Song, W. S. Cheng, R. E. Cupps, J. D. Earle, G. M. Farrow, and M. M. Lieber, "Nuclear deoxyribonucleic acid content measured by static cytometry: important prognostic association for patients with clinically localized prostate carcinoma treated by external beam radiotherapy," The Journal of Urology, vol. 147, no. 3, pp. 794-797, 1992.

[54] G. Zafarana, A. S. Ishkanian, C. A. Malloff et al., "Copy number alterations of c-MYC and PTEN are prognostic factors for relapse after prostate cancer radiotherapy," Cancer, vol. 118, no. 16, pp. 4053-4062, 2012.

[55] J. A. Locke, G. Zafarana, A. S. Ishkanian et al., "NKX3.1 haploinsufficiency is prognostic for prostate cancer relapse following surgery or image-guided radiotherapy," Clinical Cancer Research, vol. 18, no. 1, pp. 308-316, 2012.

[56] A. Berlin, E. Lalonde, J. Sykes et al., "NBN gain is predictive for adverse outcome following image-guided radiotherapy for localized prostate cancer," Oncotarget, vol. 5, no. 22, pp. 1108111090, 2014.

[57] J. A. Locke, G. Zafarana, C. A. Malloff et al., "Allelic loss of the loci containing the androgen synthesis gene, StAR, is prognostic 
for relapse in intermediate-risk prostate cancer," The Prostate, vol. 72, no. 12, pp. 1295-1305, 2012.

[58] S. J. Freedland, L. Gerber, J. Reid et al., "Prognostic utility of cell cycle progression score in men with prostate cancer after primary external beam radiation therapy," International Journal of Radiation Oncology, Biology, Physics, vol. 86, no. 5, pp. 848853, 2013.

[59] E. Lalonde, A. S. Ishkanian, J. Sykes et al., "Tumour genomic and microenvironmental heterogeneity for integrated prediction of 5-year biochemical recurrence of prostate cancer: a retrospective cohort study," The Lancet Oncology, vol. 15, no. 13, pp. 15211532, 2014.

[60] L.-Y. Khor, M. Desilvio, R. Li et al., "Bcl-2 and bax expression and prostate cancer outcome in men treated with radiotherapy in Radiation Therapy Oncology Group protocol 86-10," International Journal of Radiation Oncology Biology Physics, vol. 66, no. 1, pp. 25-30, 2006.

[61] F. A. Stewart and A. van der Kogel, "Proliferative and cellular organization of normal tissues," in Basic Clinical Radiobiology, G. G. Steel, Ed., pp. 23-29, Arnold, London, UK, 2002.

[62] J. W. Hopewell, J. Nyman, and I. Turesson, "Time factor for acute tissue reactions following fractionated irradiation: a balance between repopulation and enhanced radiosensitivity," International Journal of Radiation Biology, vol. 79, no. 7, pp. 513524, 2003.

[63] G. M. Morris and J. W. Hopewell, "Changes in the cell kinetics of pig epidermis after repeated daily doses of X rays," British Journal of Radiology. Supplement, vol. 19, pp. 34-38, 1986.

[64] I. Turesson and H. D. Thames, "Repair capacity and kinetics of human skin during fractionated radiotherapy: erythema, desquamation, and telangiectasia after 3 and 5 year's follow-up," Radiotherapy and Oncology, vol. 15, no. 2, pp. 169-188, 1989.

[65] J. Thacker and R. E. Wilkinson, "The genetic basis of cellular recovery from radiation damage: response of the radiosensitive irs lines to low-dose-rate irradiation," Radiation Research, vol. 144, no. 3, pp. 294-300, 1995.

[66] N. Somaiah, J. Yarnold, A. Lagerqvist, K. Rothkamm, and T. Helleday, "Homologous recombination mediates cellular resistance and fraction size sensitivity to radiation therapy," Radiotherapy and Oncology, vol. 108, no. 1, pp. 155-161, 2013.

[67] N. Somaiah, J. Yarnold, F. Daley et al., "The relationship between homologous recombination repair and the sensitivity of human epidermis to the size of daily doses over a 5-week course of breast radiotherapy," Clinical Cancer Research, vol. 18, no. 19, pp. 5479-5488, 2012.

[68] D. L. Rimm, "What brown cannot do for you," Nature Biotechnology, vol. 24, no. 8, pp. 914-916, 2006.

[69] D. Trudel, G. Zafarana, J. Sykes, C. L. Have, R. G. Bristow, and T. van der Kwast, "4FISH-IF, a four-color dual-gene FISH combined with p63 immunofluorescence to evaluate NKX3.1 and MYC status in prostate cancer," The Journal of Histochemistry and Cytochemistry, vol. 61, no. 7, pp. 500-509, 2013.

[70] A. Bylund, P. Stattin, A. Widmark, and A. Bergh, "Predictive value of bcl-2 immunoreactivity in prostate cancer patients treated with radiotherapy," Radiotherapy and Oncology, vol. 49, no. 2, pp. 143-148, 1998.

[71] M. Abdel-Wahab, B. A. Berkey, A. Krishan et al., "Influence of number of CAG repeats on local control in the RTOG 86-10 protocol," American Journal of Clinical Oncology, vol. 29, no. 1, pp. 14-20, 2006.

[72] M. Roach III, M. De Silvio, T. Rebbick et al., "Racial differences in CYP3A4 genotype and survival among men treated on
Radiation Therapy Oncology Group (RTOG) 9202: a phase III randomized trial," International Journal of Radiation Oncology, Biology, Physics, vol. 69, no. 1, pp. 79-87, 2007.

[73] A. S. Ishkanian, G. Zafarana, J. Thoms, and R. G. Bristow, "Array $\mathrm{CGH}$ as a potential predictor of radiocurability in intermediate risk prostate cancer," Acta Oncologica, vol. 49, no. 7, pp. 888-894, 2010.

[74] N. Erho, A. Crisan, I. A. Vergara et al., "Discovery and validation of a prostate cancer genomic classifier that predicts early metastasis following radical prostatectomy," PLoS ONE, vol. 8, no. 6, Article ID e66855, 2013.

[75] C.-L. Wu, B. E. Schroeder, X.-J. Ma et al., "Development and validation of a 32-gene prognostic index for prostate cancer progression," Proceedings of the National Academy of Sciences of the United States of America, vol. 110, no. 15, pp. 6121-6126, 2013.

[76] D. Knezevic, A. D. Goddard, N. Natraj et al., "Analytical validation of the Oncotype DX prostate cancer assay-a clinical RT-PCR assay optimized for prostate needle biopsies," BMC Genomics, vol. 14, article 690, 2013.

[77] J. Cuzick, G. P. Swanson, G. Fisher et al., "Prognostic value of an RNA expression signature derived from cell cycle proliferation genes in patients with prostate cancer: a retrospective study," The Lancet Oncology, vol. 12, no. 3, pp. 245-255, 2011.

[78] S. Yue, J. Li, S. Lee et al., "Cholesteryl ester accumulation induced by PTEN loss and PI3K/AKT activation underlies human prostate cancer aggressiveness," Cell Metabolism, vol. 19, no. 3, pp. 393-406, 2014.

[79] J. A. Watkins, S. Irshad, A. Grigoriadis, and A. N. J. Tutt, "Genomic scars as biomarkers of homologous recombination deficiency and drug response in breast and ovarian cancers," Breast Cancer Research, vol. 16, no. 3, article 211, 2014.

[80] D. Manson-Bahr, R. Ball, G. Gundem et al., "Mutation detection in formalin-fixed prostate cancer biopsies taken at the time of diagnosis using next-generation DNA sequencing," Journal of Clinical Pathology, vol. 68, no. 3, pp. 212-217, 2015.

[81] S. Carreira, A. Romanel, J. Goodall et al., "Tumor clone dynamics in lethal prostate cancer," Science Translational Medicine, vol. 6, no. 254, Article ID 254ra125, 2014. 


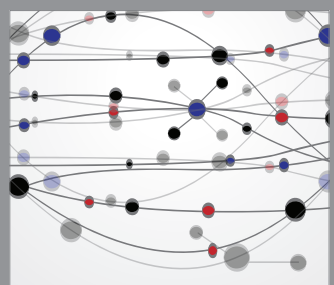

The Scientific World Journal
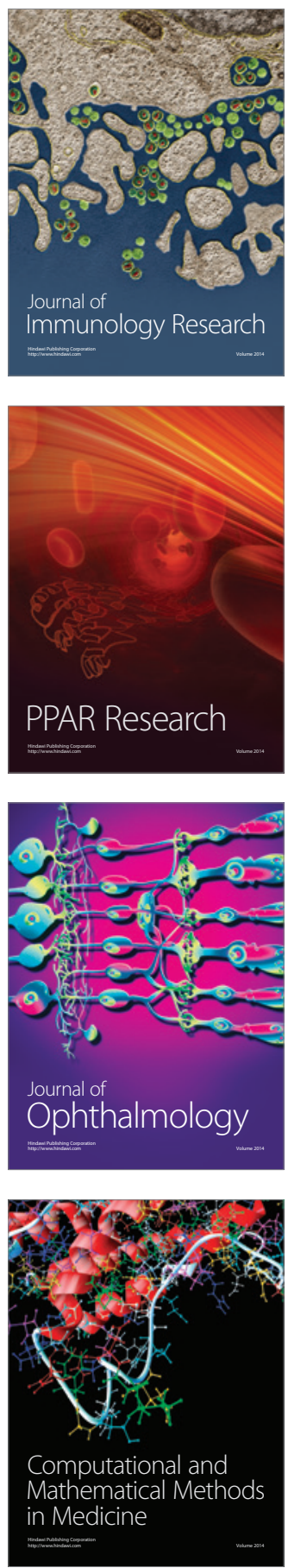

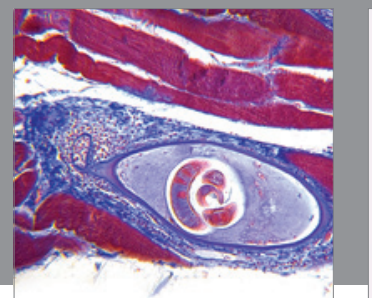

Gastroenterology

Research and Practice
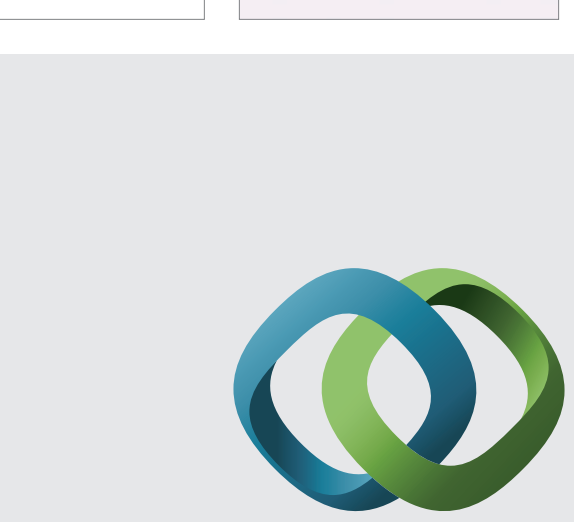

\section{Hindawi}

Submit your manuscripts at

http://www.hindawi.com
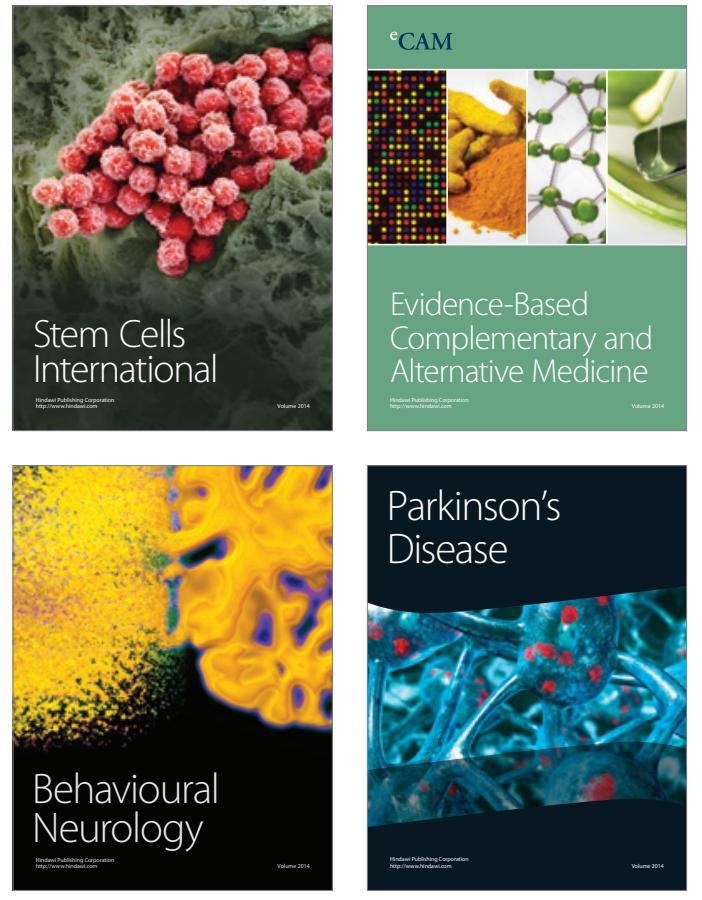
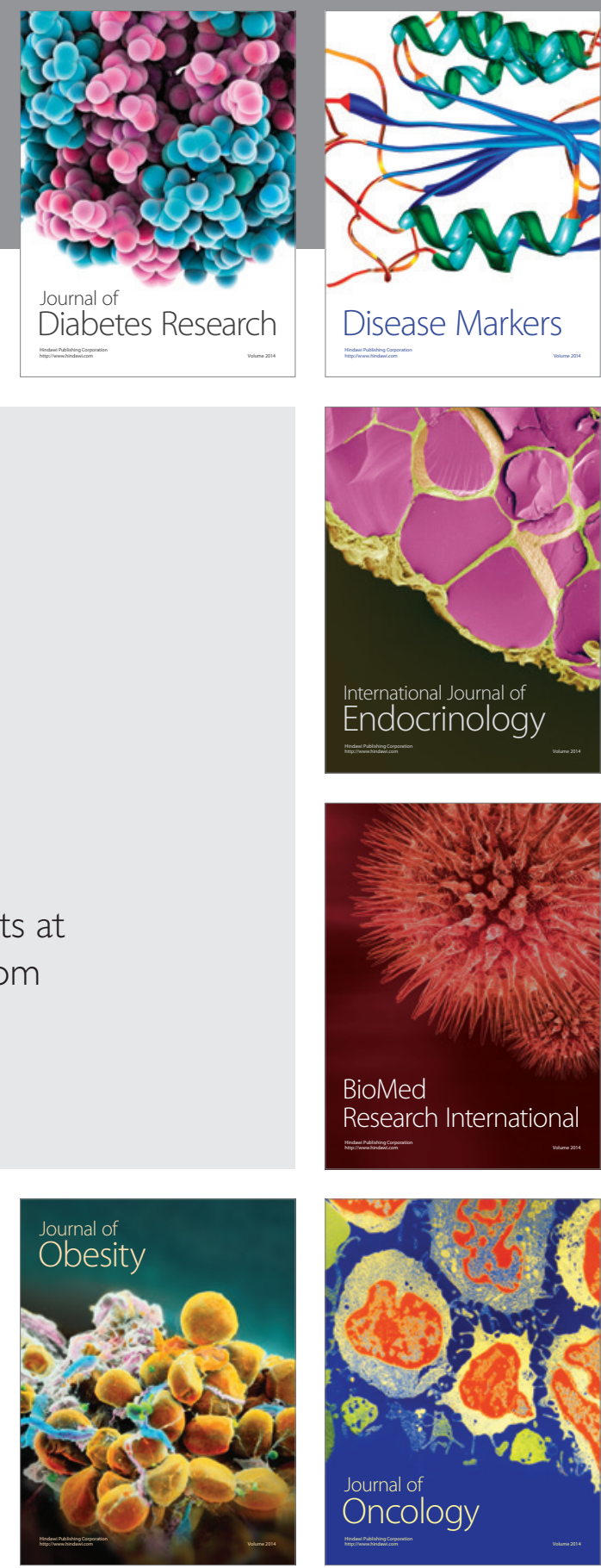

Disease Markers
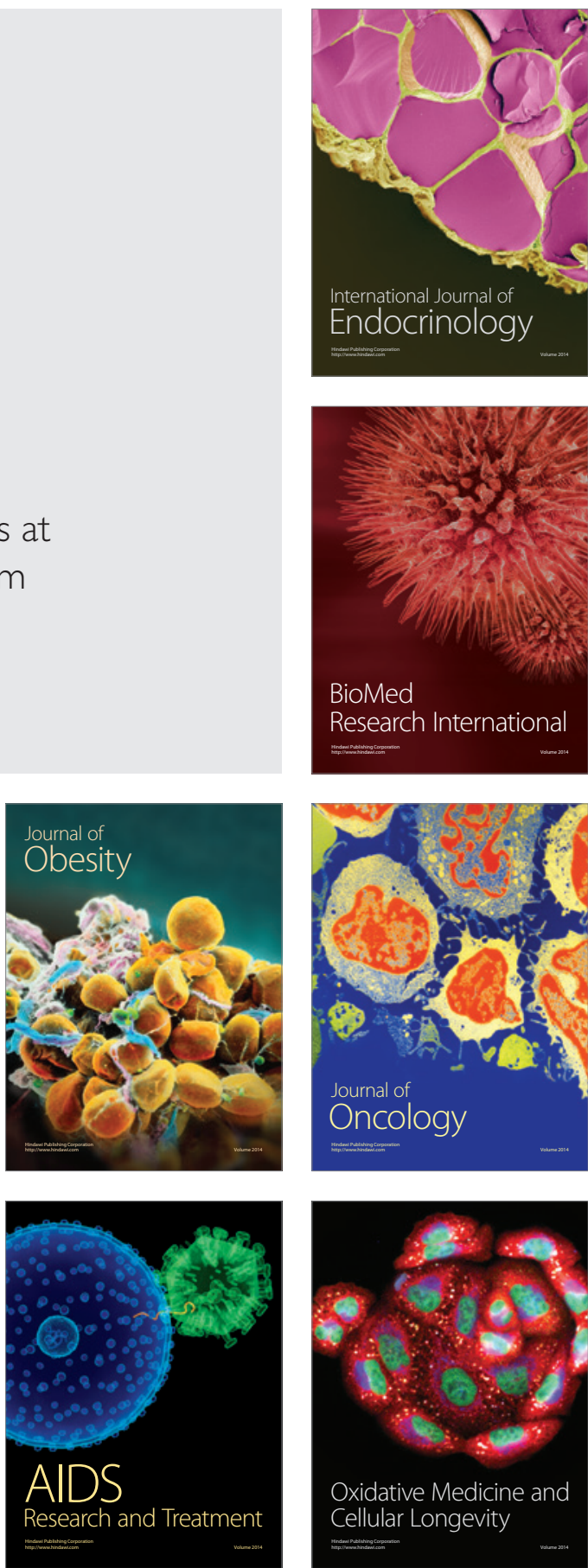